\title{
Association of Elevated Protein Kinase CK2 Activity with Aggressive Behavior of Squamous Cell Carcinoma of the Head and Neck
}

\author{
Markus Gapany,* Russell A. Faust,* Sherif Tawfic, ${ }^{+}$ \\ Alan Davis, ${ }^{*}$ George L. Adams, ${ }^{*}$ and Khalil Ahmed ${ }^{\text {t‡ }}$ \\ Departments of *Otolaryngology and Head \& Neck Surgery, and \\ 'Laboratory Medicine and Pathology, University of Minnesota, \\ Minneapolis, Minnesota, U.S.A. \\ ${ }^{\ddagger}$ Cellular and Molecular Biochemistry Research Laboratory, \\ Department of Veterans Affairs Medical Center, Minneapolis, \\ Minnesota, U.S.A.
}

\begin{abstract}
Background: Protein kinase CK2 (also known as casein kinase 2) is a messenger-independent protein serine/ threonine kinase ubiquitously distributed in eukaryotes. CK2 has been found to phosphorylate a wide variety of cytosolic and nuclear substrates which are intimately involved in regulation of DNA, RNA, and protein synthesis, and differentiation. We therefore addressed the hypothesis that malignant transformation of upper aerodigestive tract mucosa to squamous cell carcinoma of the head and neck (SCCHN) might be associated with altered CK2 activity.

Materials and Methods: To this end, we subjected surgical specimens of SCCHN tumors and of normal oropharyngeal mucosa to subcellular fractionation. We then quantitated CK2 activity in cytosol and nuclei of these specimens using a CK2-specific peptide substrate (Arg-Arg-Arg-Glu-Glu-Glu-Thr-GluGlu-Glu).
\end{abstract}

\begin{abstract}
Results: We found that CK2 activity was significantly elevated in both nuclear $(p<0.0005)$ and cytosolic $(p<0.0034)$ compartments of SCCHN tumors, relative to normal oropharyngeal mucosa. Moreover, CK2 activity in the cellular cytosolic fraction of SCCHN tumors was associated with less differentiated histologic grade $(p<$ $0.037)$, positive nodal metastatic status $(p<0.056)$, and a poor clinical outcome $(p<0.028)$. Kaplan-Meier $\mathrm{cu}$ mulative survival analysis revealed greatly reduced survival in the high-CK2 activity patient group, with high statistical significance $(p<0.023)$.

Conclusions: These preliminary data reveal that malignant transformation of the upper aerodigestive tract mucosa is associated with altered CK2 activity. The results further suggest that dysregulation of this protein kinase may play a significant role in the pathobiology of SCCHN, and that CK2 activity may be a prognostic indicator in this malignancy.
\end{abstract}

\section{INTRODUCTION}

Squamous cell carcinoma of the head and neck (SCCHN) comprises approximately $90 \%$ of the malignancies of the head and neck $(1,2)$. These are aggressive neoplasms which pose a significant international public health problem (1-3);

Address correspondence and reprint requests to: Khalil Ahmed, Cellular and Molecular Biochemistry Research Laboratory (151), VA Medical Center, One Veterans Drive, Minneapolis, MN 55417, U.S.A. in the United States alone, more than 40,000 new cases are diagnosed annually. In spite of a steady increase in incidence, and the significant morbidity and mortality carried with it, advances in treatment have not resulted in measurable improvement in overall survival; one-third of these patients die within 5 years despite multimodality therapy $(4,5)$. Although early diagnosis has been demonstrated to improve overall survival rates in these patients, proper recognition of the malignant potential of SCCHN lesions has 
been hindered by a lack of observer-independent histopathologic criteria and cellular markers of aggressive tumor behavior (6-8). An improved understanding of the biological aggressiveness, or malignant potential, of a particular tumor at the time of presentation would allow for prudent choices among treatment options, and would ideally contribute to therapeutic advancements. While some biomarkers of malignant transformation in SCCHN have been advanced, none have yet proven to be clinically useful (7-13).

Recent advances in the field of regulatory protein phosphorylation may provide a promising area for clinical research focussed on potential prognostic markers. Phosphorylation of proteins as a post-translational modification is considered to play a pivotal role in the regulation of cellular growth and differentiation (reviewed in Refs. 14-16). Aberrant phosphorylation of a variety of regulatory proteins, including oncogene products, and kinases themselves, has been associated with malignant transformation in several cancers (reviewed in Ref. 17). In cancers other than SCCHN, kinase activation is clearly associated with malignant transformation; protein kinase CK2 is one such kinase.

CK2 is a ubiquitous serine/threonine kinase known to occur as a tetrameric complex of $\alpha(\mathrm{Mr}$ $41,000), \alpha^{\prime}(\operatorname{Mr~38,000)}$, and $\beta(\operatorname{Mr~28,000)}$ subunits, with an $\alpha_{2} \beta_{2}$ or $\alpha \alpha^{\prime} \beta_{2}$ configuration, and is localized in both cytosolic and nuclear subcellular compartments (reviewed in Ref. 18). CK2 is a multipotential enzyme, and is reported to phosphorylate a number of substrates known to play a pivotal role in cell division and differentiation (18). There is evidence that CK2 may transmit signals to the nucleus by its translocation between the cytoplasm and the nucleus in response to growth stimuli (19-21). Furthermore, several reports suggest that $\mathrm{CK} 2$ activity is modulated by various agents, including steroid hormones, insulin, and epidermal growth factor (EGF) $(18,19,22,23)$. CK2 activation has been associated with malignant transformation. As examples, elevated levels of CK2 activity have been detected in human prostatic, lung, and colon carcinomas $(19,24,25)$. The role of CK2 as an oncogene is further supported by a recent report that overexpression of CK2 in the lymphocytic compartment of transgenic mice is capable of transforming lymphocytes (26).

We therefore addressed the hypothesis that malignant transformation of upper aerodigestive tract mucosa to SCCHN may be associated with altered CK2 activity. Furthermore, we postulated that altered subcellular distribution of CK2 might be associated with biological behavior of SCCHN. To this end, in this pilot cohort study we quantitated CK2 activity in the cytoplasmic and nuclear subcellular compartments of 20 surgical SCCHN tumor specimens, as well as in subcellular fractions of normal upper aerodigestive tract mucosa. We evaluated activity of this protein kinase in relation to clinical stage, histopathological grade, metastatic status of the tumors, and cumulative patient survival analysis. Our preliminary results hint that CK2 may be a marker of malignant transformation from normal oropharyngeal mucosa to SCCHN, and that its activity may be associated with aggressive tumor behavior.

\section{MATERIALS AND METHODS}

\section{Patients and Tumor Characteristics}

A cohort of 20 patients treated surgically for SCCHN at the Minneapolis Veterans Affairs

TABLE 1. Patient and tumor characteristics

\begin{tabular}{lr}
\hline Patient $^{a}$ and Tumor Characteristics & No. \\
\hline Gender & 19 \\
Male & 1 \\
Female & \\
Tumor Site & 4 \\
Larynx & 2 \\
$\quad$ Supraglottic & 4 \\
$\quad$ Glottic & \\
Pyriform sinus & 3 \\
Oral cavity & 2 \\
$\quad$ Oral tongue & 2 \\
$\quad$ Floor of mouth & \\
$\quad$ Retromolar trigone & 2 \\
Oropharynx & 18 \\
$\quad$ Tonsil & 1 \\
$\quad$ Base of tongue & \\
Tumor differentiation & 7 \\
Poor & 11 \\
Moderate & 2 \\
Well & \\
Treatment & \\
Surgery alone & \\
Surgery + radiation & \\
\hline & \\
&
\end{tabular}

$a_{n}=20 ;$ median age $=63$ years (range: $41-73$ years). 
Medical Center between July 1992 and January 1993 were prospectively evaluated in this study. All patients presented with a single primary tumor, and none had undergone any preoperative treatment. As shown in Table l, there were 19 males and 1 female, their ages ranging from 41 to 73 years (mean 62.7 years). Tumor specimens were obtained from four different head and neck sites including larynx, pyriform sinus, oral cavity, and oropharynx. Moderately differentiated tumors were predominant; all four tumor stages were included. At the time of surgical resection, 20 specimens of SCCHN were procured intraoperatively and immediately frozen in liquid nitrogen; specimens were stored in liquid nitrogen until the time of evaluation. Histological confirmation of the tumor was carried out by light microscopy. All except two patients underwent surgical resection of the tumor with neck dissection followed by postoperative radiotherapy (Patients 3 and 10 received no postoperative radiation therapy). The follow-up ranged from 21 to 32 months. In addition to the SCCHN tumors, six specimens of normal upper aerodigestive tract mucosa were obtained in the operating room from adult nonsmokers/nondrinkers undergoing tonsillectomy and uvulopalatoplasty, and these specimens were treated in as the tumors were.

\section{Preparation of Cytosolic and Nuclear Subcellular Fractions}

Cytosolic and nuclear fractions from both normal mucosa and SCCHN tumors were prepared as follows: tissue specimens were minced and homogenized in $0.32 \mathrm{M}$ sucrose, $3 \mathrm{mM} \mathrm{MgCl}_{2}, 5 \mathrm{mM}$ 2-mercaptoethanol, $0.5 \mathrm{mM}$ phenylmethylsulfonyl fluoride (PMSF), $5 \mu \mathrm{g} / \mathrm{ml}$ of aprotinin, and 1 $\mu \mathrm{g} / \mathrm{ml}$ of leupeptin, to yield a $10 \%(\mathrm{w} / \mathrm{v})$ homogenate. The tissue homogenate was centrifuged for $5 \mathrm{~min}$ at $600 \times \mathrm{g}$ to obtain a crude nuclear pellet. The supernatant containing the cytosolic fraction was collected, cleared by centrifugation at $100,000 \times g$ for $60 \mathrm{~min}$, and aliquots of the supernatant phase were stored at $-20^{\circ} \mathrm{C}$. The pellet containing the crude nuclei was suspended in a medium consisting of $20 \mathrm{mM}$ HEPES pH 7.9, $1 \mathrm{mM}$ EDTA, $1 \mathrm{mM}$ EGTA, $1 \mathrm{mM}$ dithiothreitol, $10 \%$ (v/v) glycerol, $0.02 \%$ Tween-20, $0.25 \mathrm{mM}$ PMSF, $5 \mu \mathrm{g} / \mathrm{ml}$ aprotinin, $1 \mu \mathrm{g} / \mathrm{ml}$ leupeptin (HETED buffer) containing $0.4 \mathrm{M} \mathrm{KCl}$. Following a 30 -min incubation on ice, the material was centrifuged at $100,000 \times g$ for $60 \mathrm{~min}$. The supernatant of extracted soluble nuclear proteins was then dialyzed overnight at $4^{\circ} \mathrm{C}$ against HETED buffer containing $0.1 \mathrm{M} \mathrm{KCl}$. The dialyzed material was centrifuged at $25,000 \times g$ for $20 \mathrm{~min}$ and the supernatant stored at $-20^{\circ} \mathrm{C}$.

\section{Quantitation of CK2 Activity}

Protein kinase CK2 activity in nuclear extracts and cytosolic fractions was measured using the specific CK2 synthetic decapeptide substrate, [Arg-Arg-Arg-Glu-Glu-Glu-Thr-Glu-Glu-Glu], as described (21). In brief, the reaction medium consisted of $30 \mathrm{mM}$ Tris- $\mathrm{HCl}, \mathrm{pH} 7.4,5 \mathrm{mM}$ $\mathrm{MgCl}_{2}, 1 \mathrm{mM}$ dithiothreitol, $150 \mathrm{mM} \mathrm{NaCl}, 0.05$ $\mathrm{mM}\left[\gamma^{32} \mathrm{P}\right]-\mathrm{ATP}$, and $1 \mathrm{mM}$ decapeptide substrate, in a final volume of $100 \mu \mathrm{l}$. The reaction was started by adding CK2 source (cytosolic, or soluble nuclear protein sample) containing 2-5 $\mu \mathrm{g}$ of protein, and was carried out over a time course of 0 to $30 \mathrm{~min}$ at $37^{\circ} \mathrm{C}$. Under these conditions, the reaction rate was linear with respect to time and amount of the enzyme. At appropriate times, aliquots were placed on Whatman P-81 paper, and the reaction was stopped by placing the papers in $10 \mathrm{mM}$ phosphoric acid. Unreacted $\left[\gamma^{32} \mathrm{P}\right]$-ATP was removed from the papers by washing them twice in phosphoric acid followed by two washes in $15 \%$ acetic acid, then acetone. The amount of ${ }^{32} \mathrm{P}$ incorporated into the CK2 substrate peptide was determined using liquid scintillation spectrometry. CK2 activity was expressed as nanomoles of ${ }^{32} \mathrm{P}$ transferred per hour per milligram protein.

\section{Statistical Analysis of CK2 Activity}

Differences between patient groups were compared using the chi-square test. The cumulative survival curves for patients groups were calculated by the Kaplan-Meier method, compared using the generalized Wilcoxon test, and relative risk-ratios were calculated by the Cox method (27).

\section{RESULTS \\ CK2 Activities in Subcellular Compartments}

Using the synthetic, CK2-specific decapeptide substrate, we first quantitated CK2 activity in cytosolic and soluble nuclear fractions of normal oropharyngeal mucosa specimens. We found that CK2 activity in the soluble nuclear fraction 
TABLE 2. Tumor characteristics

\begin{tabular}{ccccccc}
\hline & & & & & \multicolumn{2}{c}{ CK2 Activity $^{a}$} \\
\cline { 5 - 7 } No. & & & & & & \\
\cline { 4 - 6 } & TNM & Differentiation & Nodes $^{b}$ & Outcome & Cytosol & Nucleus \\
\hline 1 & T2N2 & Poor & Pos. & DOD & 9.1 & 19.8 \\
2 & T3N1 & Mod. & Pos. & AFOD & 4.6 & 16.9 \\
3 & T1N0 & Poor & Neg. & AFOD & 3.9 & 13.6 \\
4 & T3N0 & Mod. & Neg. & AFOD & 3.2 & 7.2 \\
5 & T4N2 & Poor & Pos. & DOD & 6.8 & 6.8 \\
6 & T3N2 & Poor & Pos. & AFOD & 7.3 & 53.4 \\
7 & T3N0 & Mod. & Neg. & AFOD & 5.0 & 18.0 \\
8 & T3N0 & Mod. & Neg. & AFOD & 2.1 & 9.2 \\
9 & T4N3 & Mod. & Pos. & DOD & 8.2 & 41.9 \\
10 & T2N0 & Mod. & Neg. & AFOD & 1.0 & 17.7 \\
11 & T1N3 & Poor & Pos. & DOD & 5.9 & 29.3 \\
12 & T3N0 & Mod. & Neg. & AFOD & 8.3 & 24.0 \\
13 & T3N0 & Well & Neg. & AFOD & 4.1 & 19.5 \\
14 & T4N2 & Well & Pos. & DOD & 1.4 & 5.7 \\
15 & T4N0 & Mod. & Neg. & AFOD & 3.6 & 8.9 \\
16 & T4N0 & Mod. & Neg. & AFOD & 5.2 & 37.0 \\
17 & T4N0 & Poor & Pos. & DOD & 15.4 & 36.7 \\
18 & T4N2 & Mod. & Pos. & DOD & 6.8 & 26.4 \\
19 & T2N1 & Poor & Pos. & AFOD & 4.6 & 20.0 \\
20 & T4N0 & Mod. & Pos. & AFOD & 4.6 & 31.6 \\
Mean $($ SD) & & & & & $5.9(3.5)$ & $20.3(10.4)$ \\
& & & & & & \\
\hline
\end{tabular}

${ }^{a} \mathrm{CK} 2$ activity presented as nanomoles ${ }^{32} \mathrm{P}$ transferred per hour per milligram protein in cytosolic or nuclear samples assayed.

${ }^{b}$ Nodal status was determined by histopathological evaluation of the neck dissection specimens for each case.

(3.7 nmoles $/ \mathrm{hr} / \mathrm{mg}$ protein) was significantly greater than in the cytosolic compartment $(0.6$ nmoles/hr/mg protein). We next quantitated CK2 in subcellular fractions of SCCHN tumor specimens. As shown in Table 2, CK2 activity in SCCHN tumors was significantly elevated in both the nuclear (mean $=20.3 ; p<0.0005)$ and cytosolic (mean $=5.9 ; p<0.0034$ ) compartments relative to normal mucosa. The relationship between CK2 activity in cytosol and nuclear fractions of SCCHN tumors and normal mucosa is apparent in Fig. 1.

\section{Association of CK2 Expression with Clinicopathologic Parameters}

The observation that both cytosolic and nuclear levels of CK2 activity were significantly elevated in SCCHN tumors (Fig. 1) prompted us to exam-
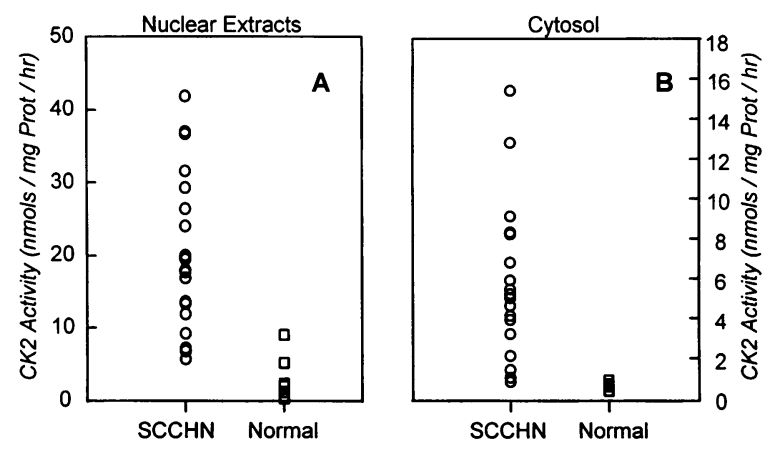

FIG. 1. CK2 activity in squamous cell carcinoma of the head and neck versus normal mucosa

CK2 activity was measured in subcellular fractions of 20 SCCHN tumors $(O)$ and in six specimens of normal upper aerodigestive tract mucosa ( $\square$ ): CK2 activity in SCCHN subcellular fractions was significantly elevated in both nuclear (20.3 versus 3.7; $p<0.0005)$ and cytosolic ( 5.9 versus $0.6 ; p<0.0034$ ) compartments relative to normal mucosa. (A) CK2 activity in soluble nuclear proteins. (B) CK2 activity in cytosol. 


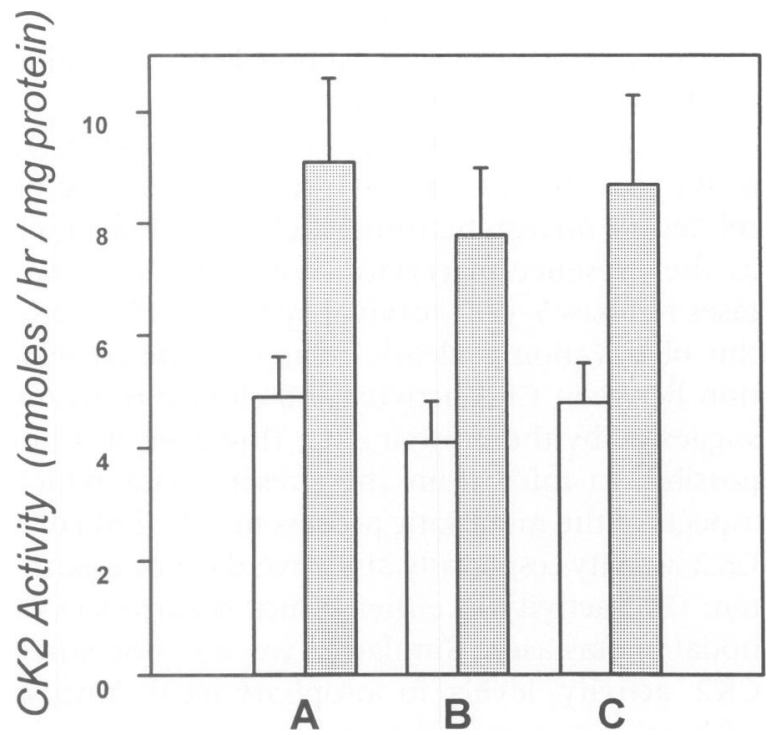

FIG. 2. Clinicopathologic correlates of CK2 activity in squamous cell carcinoma of the head and neck

Clinicopathologic correlates of CK2 activity in cytosolic fractions of SCCHN. CK2 activity was measured in cytosolic fractions of SCCHN tumors using the CK2-specific decapeptide substrate, and activity presented as nanomoles ${ }^{32} \mathrm{P}$ transferred to substrate per hour incubation per milligram protein in sample, as described in Materials and Methods. (A) CK2 activity is displayed by histopathologic differentiation: moderate differentiation ( $n=11$; open bar) vs. poor differentiation ( $n=7$; shaded bar); $p<0.037$. Welldifferentiated tumors were not included in the analysis due to low $n(n=2)$. (B) CK2 activity displayed by nodal status: node-negative $(n=9$; open bar) versus node-positive ( $n=11$; shaded bar); $p<$ 0.056 . (C) CK2 activity displayed by clinical outcome: alive free of disease $(n=13$; open bar) versus dead of disease ( $n=7$; shaded bar); $p<0.028$. All data are plotted as the mean \pm SEM.

ine CK2 activities in these samples in relation to the clinical and histopathological tumor parameters, including stage of disease, nodal status, degree of tumor differentiation, and patient survival. These data, provided in Table 2, point to several correlations. First, cytosolic CK2 activity in SCCHN tumors was significantly correlated $(p<0.037)$ with poorer histological differentiation (Fig. 2A). Second, higher cytosolic CK2 activity was associated $(p<0.056)$ with metastatic behavior: those tumors having histopathologically proven lymph node metastases exhibited higher cytosolic CK2 activity than those that were negative for metastases (Fig. 2B). Furthermore, tumors from patients who were dead of

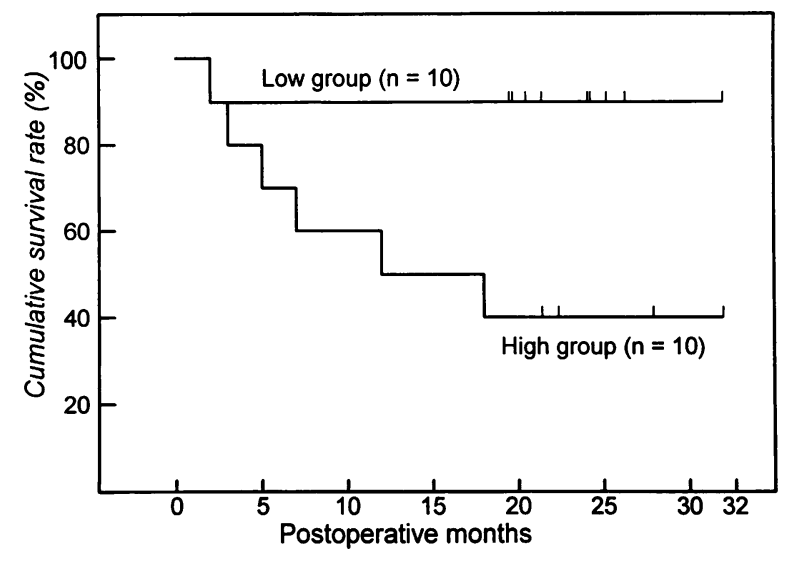

FIG. 3. Cumulative survival curves based on CK2 levels

The median value of 4.8 for cytosolic CK2 activity in primary SCCHN tumors was used to divide patients into High-CK2 and Low-CK2 activity groups. The cumulative survival curves for the two groups were calculated by the Kaplan-Meier method. Chi-square analysis reveals a statistical significance between the two groups of $p<0.023$.

disease (DOD) within two to eighteen months of diagnosis exhibited significantly higher cytosolic CK2 activity $(p<0.028)$ than tumors from patients who are alive and free of disease (AFOD) on follow-up at 20 to 32 months, as illustrated in Fig. 2C. In this study population, we had no patients who were alive with disease/recurrence.

When patients were divided into two groups based on the median cytosolic CK2 activity (4.8), patients in the Low-CK2 activity group exhibited significantly greater cumulative survival than those patients in the High-CK2 activity group $(p<0.023)$. Cox survival analysis revealed a relative risk-ratio for SCCHN tumor-cytosolic CK2 activity of 1.43. The Kaplan-Meier analysis of these data is illustrated in Fig. 3.

In contrast to the above associations, CK2 activity in the soluble nuclear fraction of SCCHN tumor samples revealed no significant relation to any of the examined clinicopathological parameters in this series. Although Kaplan-Meier survival analysis of CK2 activity in the soluble nuclear fraction of SCCHN tumors did suggest a trend similar to that observed for cytosolic CK2 activity, this was not statistically significant $(p=$ $0.16)$. Similarly, there was no significant correlation between clinical (TNM) stage and CK2 activity evident in either cytosolic or nuclear compartments of SCCHN. 


\section{DISCUSSION}

The purpose of this study was to better understand the role of CK2 expression in relation to head and neck tumor clinicopathologic behavior. To this end, we have quantitated the activity of CK2, a cellular protein kinase which is implicated in cell growth and differentiation $(18,19,26)$. Comparing CK2 activity in the subcellular compartments of both normal mucosa of nondrinkers/nonsmokers and SCCHN revealed several interesting correlations. CK2 activity levels in both cytosolic and nuclear soluble protein fractions from SCCHN were significantly elevated compared with those in normal mucosa. Furthermore, the data suggest an association between CK2 activity in SCCHN cytosolic fractions and histologic grade $(p<0.037)$, nodal status of individual patients $(p<0.056)$, and, within the short 21-to 32-month follow-up of this study, with clinical outcome-those patients with the highest levels of CK2 activity in the cytosolic fractions of their SCCHN tumors are deceased at the time of this writing $(p<0.028)$. These findings suggest that CK2 may be useful as an observer-independent marker of histological differentiation, and although preliminary, these results hint that CK2 activity in SCCHN may be related to increased biological aggressiveness of these tumors. The Kaplan-Meier survival data suggest that cytosolic CK2 activity may be a significant prognostic indicator in SCCHN. Association between CK2 activity and biological behavior of malignant tumors has not been previously investigated, and its value as a prognostic indicator remains to be validated in a larger prospective clinical series. Regardless, these findings may be of major clinical significance.

Elevated CK2 activity in cell lysates, nuclear preparations, or crude tissue homogenates of prostate, colon, and lung carcinomas have been reported $(19,24,25)$, but correlation of CK2 activity in subcellular compartments with clinicopathologic parameters has not been previously examined in detail. The present data provide the first account of the subcellular compartmentation of CK2 activity in human tumors, and the association of CK2 activity within subcellular compartments with tumor behavior. Interestingly, although the nuclear fractions of SCCHN tumors exhibited the highest CK2 activities, the data did not reveal a significant correlation between these nuclear activities and clinicopathological parameters. Confirmation of the subcellular localization of CK2 in SCCHN using immunohisto- chemical methods is currently under way, and further investigation into the subnuclear localization of CK2 in SCCHN seems warranted.

These initial data suggest that cytosolic CK2 activity in the primary SCCHN tumors may be related to metastatic tumor behavior. Inasmuch as the presence of cervical lymph node metastases reduces 5 -year survival by nearly $45 \%(28)$, this observation is clearly related to the correlation between CK2 activity and clinical outcome suggested by the present data. However, it is not possible to infer from the present data which aspects of the metastatic process may be linked to CK2 activity, especially since we did not quantitate CK2 activity in either tumor recurrences or nodal metastases. Similarly, we did not study CK2 activity levels in oropharyngeal mucosa with respect to staged tumor progression per se (hyperplasia through carcinoma), as has been described for expression of proliferating cell nuclear antigen (9) and EGF receptors (10) in the multistep process of SCCHN tumorigenesis; follow-up studies of CK2 in these tissues are indicated. Finally, it may promote our understanding of the pathogenesis of this malignancy to determine whether the elevated levels of CK2 activity detected in SCCHN reflect transcriptional, translational, or post-translational levels of activation.

In summary, the data presented here, although preliminary, suggest that the Protein Kinase CK2 is activated with malignant transformation of upper aerodigestive tract mucosa to SCCHN, and that CK2 may play a significant role in the pathobiology of SCCHN. Investigation into the molecular basis of these novel findings, as well as follow-up study of CK2 in additional tissues, seems warranted, as these questions bear directly on the relationship between this protein kinase and SCCHN tumorigenesis.

\section{ACKNOWLEDGMENTS}

The authors wish thank biostatistician, Dr. Bruce Lindgren, for assistance with statistical design and analyses, and David Lambert for his technical assistance. This work was supported in part by the American Academy of Otolaryngology, Head \& Neck Surgery Research Training Award (ARTA), the Medical Research Fund of the U.S. Department of Veterans Affairs, and NCI Research Grant CA-15062. Portions of this work were presented at the Annual Meeting of the American Association for Cancer Research, 
1995, and at the American Society for Head and Neck Surgery 36th Annual Meeting, 1994.

\section{REFERENCES}

1. Devesa SS, Silverman DT, Young JL, et al. (1987) Cancer incidence and mortality trends among whites in the United States 1947-1984. J. Natl. Cancer Inst. 4: 701-770.

2. Silverberg E, Boring CC, Squires T. (1990) Cancer statistics, 1990. C.A. Cancer J. Clin. 40: 9-26.

3. Voravud N. (1990) Cancer in the Far East. In: Sikara K, Halnan KE (eds). Treatment of Cancer. 2nd Ed. Chapman and Hall Medical, London, pp. 887-894.

4. Batsakis JG. (1990) Squamous cell carcinomas of the oral cavity and the oropharynx. In: Batsakis JG (ed). Tumors of the Head and Neck: Clinical and Pathological Considerations. 2nd Ed. Williams \& Williams, Baltimore, MD, pp. 144-176.

5. Wolf GT, Lippman SM, Laramore G, Hong WK. (1990) Head and neck cancer. In: Halland JF, Frei E, Bast Jr RC, Kufe DW, Morton DC, Weischelbaum R (eds). Cancer Medicine. 3rd Ed., Lea and Febiger, Philadelphia, pp. 887-894.

6. Crissman JD, Zarbo RJ. (1989) Dysplasia, in situ carcinoma and progression to invasive squamous cell carcinoma of the upper aerodigestive tract. Am. J. Surg. Pathol. 13: 5-16.

7. Walther EK, Dahlmann N, Gorgulla HT. (1993) Tumor markers in the diagnosis and follow-up of head and neck cancer: Role of CEA, CA 19-9, CSS, TK, and DTTPase. Head Neck 15: 230-235.

8. Coltrera MD, Zarbo RJ, Sakr WA, Gown AM. (1993) Markers for dysplasia of the upper aerodigestive tract: Suprabasal expression of PCNA, p53, and CK19 in alcohol-fixed, embedded tissue. Am. J. Pathol. 141: 817-825.

9. Shin DM, Voravud N, Ro JY, Lee SJ, Hong WK, Hittleman WN. (1993) Sequential increases in proliferating nuclear antigen expression in head and neck tumorigenesis: A potential biomarker. J. Natl. Cancer Inst. 85: 971-978.

10. Shin DM, Ro JY, Hong WK, Hittleman WN. (1994) Dysregulation of epidermal growth factor receptor expression in premalignant lesions during head and neck tumorigenesis. Cancer Res. 54: 3153-3159.

11. Santini J, Formento JL, Francoual M, et al.
(1991) Characterization, quantification, and potential clinical value of the epidermal growth factor receptor in head and neck squamous cell carcinomas. Head Neck 13: 132-139.

12. Scambia G, Panici PB, Battaglia F, et al. (1991) Receptors for epidermal growth factor and steroid hormones in primary laryngeal tumors. Cancer 67: 1347-1351.

13. Wolf GT, Carey TE, Schmaltz SP. (1990) Altered antigen expression predicts outcome in squamous cell carcinoma of the head and neck. J. Natl. Cancer Inst. 82: 1566-1572.

14. Hunter T, Karin M. (1992) The regulation of transcription by phosphorylation. Cell 70: 375-387.

15. Pelech SL, Sanghera JS, Daya-Makin M. (1990) Protein kinase cascades in meiotic and mitotic cell cycle control. Biochem. Cell Biol. 68: 1297-1330.

16. Cantley LC, Auger KR, Carpenter C, et al. (1991) Oncogenes and signal transduction. Cell 64: 281-302.

17. Aaronson SA. (1991) Growth factors and cancer. Science 245: 1146-1153.

18. Issinger O-G. (1993) Casein kinases: Pleiotropic mediators of cellular regulation. Pharmacol. Ther. 59: 1-30.

19. Ahmed K. (1994) Significance of the casein kinase system in cell growth and proliferation with emphasis on studies of the androgenic regulation of the prostate. Cell. Mol. Biol. Res. 40: 1-11.

20. Tawfic S, Ahmed K. (1994) Growth stimulus-mediated differential translocation of casein kinase 2 to the nuclear matrix. J. Biol. Chem. 269: 24615-24620.

21. Ahmed K, Yenice S, Davis A, Goueli SA. (1993) Association of casein kinase 2 with nuclear chromatin in relation to androgenic regulation of rat prostate. Proc. Natl. Acad. Sci. 90: $4426-4430$.

22. Lorenz P, Pepperkok R, Ansorge W, Pyerin W. (1993) Cell biological studies with monoclonal and polyclonal antibodies against human casein kinase II subunit $\beta$ demonstrate participation of the kinase in mitogenic signaling. J. Biol. Chem. 268: 2733-2739.

23. Sommercorn J, Mulligan A, Lozeman FJ, Krebs E. (1987) Activation of casein kinase II in response to insulin and to epidermal growth factor. Proc. Natl. Acad. Sci. 84: 88348838.

24. Daya-Makin M, Sanhera JS, Mogentale TL, et al. (1994) Activation of a tumor-associ- 
ated protein kinase (p40TAK) and Casein Kinase 2 in human squamous cell carcinomas and adenocarcinomas of the lung. Cancer Res. 54: 2262-2269.

25. Munstermann U, Fritz G, Seitz G, Yiping L, Schneider HR, Issinger O-G. (1990) Casein kinase II is elevated in solid human tumors and rapidly proliferating non-neoplastic tissues. Eur. J. Biochem. 189: 251-257.

26. Seldin DC, Leder P. (1995) Casein kinase II $\alpha$ transgene-induced murine lymphoma: Relation to Theileriosis in cattle. Science 267: 894-897.

27. Lee ET. (1980) Statistical Methods for Survival Data Analysis. Ist Ed. Wadsworth, Belmont, CA, pp. 75-121.

28. Snow GB, Annyas AA, van Slooten EA, Bartelink H, Hart AA. (1982) Prognostic factors of neck node metastasis. Clin. Otolaryngol. 7: 185-192.

Contributed by P. Leder on July 3, 1995. 